\title{
Obstructed Direct Inguinal Hernia!: A Rare Encounter Revisited.
}

\author{
${ }^{1}$ Rahul Singh R, ${ }^{2}$ Naveed Ahmed Khan, ${ }^{3}$ Srinivasan D, ${ }^{4}$ Sreeramulu P.N, \\ ${ }^{5}$ Lakshmi Reddy \\ Department of General Surgery, SDUMC, Karnataka 563101, India
}

\begin{abstract}
Direct inguinal hernias are less likely to present as incarceration or strangulation as compared to indirect inguinal hernia, because they have wide neck. We present a case of an 80-year-old male presented with irreducible and painful swelling over the right inguinal region and features of intestinal obstruction. On exploration the obstructed hernia was found to be a direct type with gangrenous sac wall containing congested extra peritoneal pad of fat, cord structures, even the right testes was gangrenous. After gaining viability the content was reduced, posterior wall defect was closed and modified Bassini's repair was done with a right orchidectomy. A long standing direct inguinal hernia may present as acute or sub acute intestinal obstruction especially in elderly patients. Therefore, we should repair direct inguinal hernias on an elective basis in any age group.
\end{abstract}

Keywords: direct inguinal hernia, strangulation, Bassini's repair, orchidectomy

\section{Introduction}

Seventy-five percent of all abdominal wall hernias are found in the groin, making it the most common location for an abdominal wall hernia ${ }^{1}$. Of all groin hernias, $95 \%$ are hernias of the inguinal canal with the remainder being femoral hernia defects ${ }^{2}$. Two-thirds of the inguinal hernias are indirect and the remainders are direct inguinal hernia. An indirect inguinal is the most common hernia, regardless of gender. In men, indirect hernias predominate over direct hernias at a ratio of $2: 1$. Direct hernias are uncommon in women ${ }^{1}$. Direct inguinal hernias are less likely to present as incarceration or strangulation as compared to indirect inguinal hernia, because they have wide neck. The lifetime risk of inguinal hernia is $27 \%$ in men and $3 \%$ in women ${ }^{3}$. There is clearly an association between age and hernia diagnosis. After an initial predominance in infancy, groin hernias become more prevalent as the age advances. In the same way, the complications of hernias (incarceration, strangulation, and bowel obstruction) are found more commonly at the extremes of age group ${ }^{4}$. Illiteracy, financial crisis are few among many which prevent these patients from seeking medical advice.

\section{Case Report}

An 80-year-old male presented in emergency with a swelling over the right groin for 20 years which was reducible and became irreducible, painful for last eight days followed by obstipation generalized weakness, malaise loss of appetite for last four days. General physical examination and vitals of the patient was within normal limits except for mild pallor. On local examination of the right inguinal region there was a globular swelling of size $4 \times 4 \mathrm{~cm}$ present above and medial to right pubic tubercle. The swelling was globular in shape, had smooth surface, well defined margin, firm in consistency, irreducible, tender with local raise of temperature, with a negative cough impulse and a resonant note on percussion. The examination of the right testes, epididymis, spermatic cord and contralateral inguino-scrotal region was within normal limits. There was mild distension of the abdomen with diffuse tenderness, sluggish bowel sound and poor abdominal wall muscle tone. Per rectal and other systemic examination was normal. X-ray of the abdomen in erect posture though showed no air fluid levels, clinically the patient was highly suspicious for features of obstruction (figure 1). USG abdomen revealed the presence of right sided inguinal hernia containing bowel loops with very minimal fluid collection. So a diagnosis of right sided obstructed inguinal hernia was made on the basis of clinical and radiological finding. The patient was planned for surgery on an emergency basis.

A right sided inguinoscrotal skin incision was given. To our greatest surprise gangrenous hernia sac wall with pre peritoneal fat was found (Figure 2). The sac was opened and content being the congested fat with even the cord structures(Figure 3) and the testes (Figure 4) being involved. The contents were reduced after gaining vascularity and peristalsis. Excision of sac with pre- peritoneal fat was done. Excision of the gangrenous cord structures with right sided orchidectomy was done. Posterior wall defect was closed and modified Bassini's repair was done with (1-0) polypropylene interrupted suture. Post-operative period was uneventful. Patient was kept nil per oral for three days, passed flatus on third postoperative day and stool on fifth postoperative day. Patient was discharged with advice on eighth postoperative day. During the follow-up visit after three months the operation scar was found to have healed well and the patient was completely asymptomatic. 


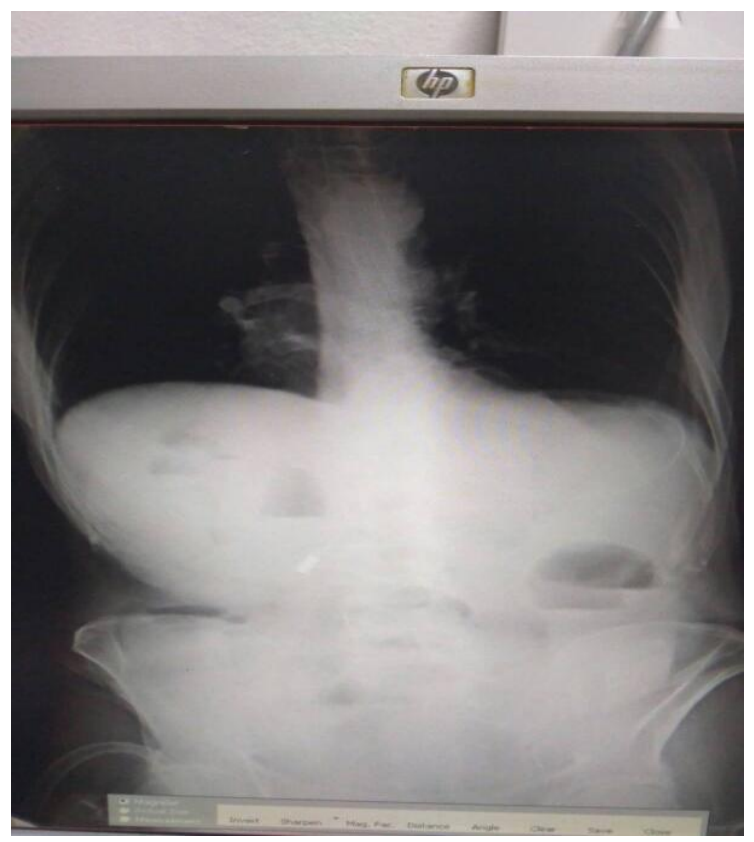

Fig 1: Erect $X$-ray of the abdomen.

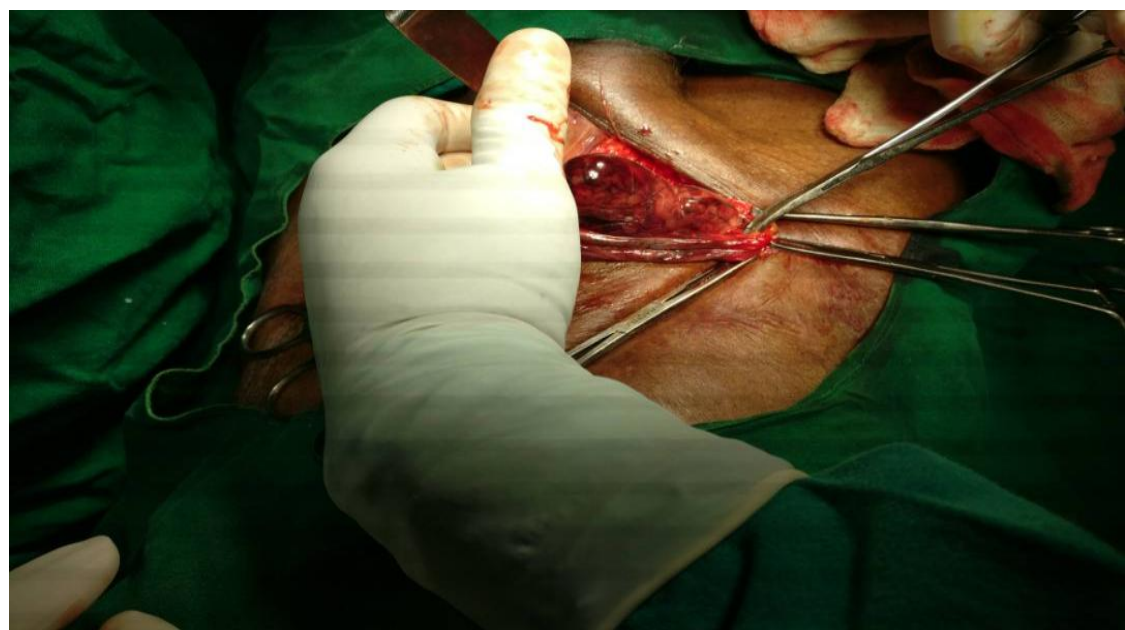

Fig 2: Gangrenous Hernial Sac with preperitoneal fat.

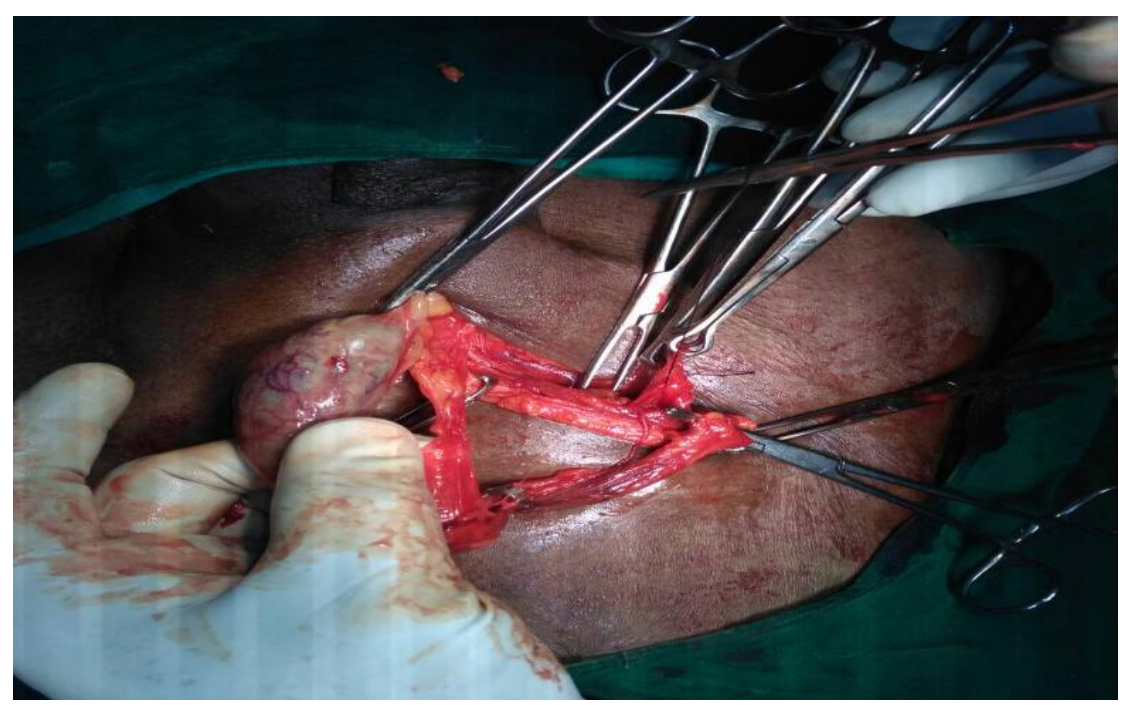

Fig 4: Gangrenous testes. (Orchidectomy was done) 


\section{Discussion}

Groin hernias are generally classified as inguinal (indirect and direct) and femoral based on the site of herniation relative to surrounding structures. Indirect hernias protrude lateral to the inferior epigastric vessels, through the deep inguinal ring.

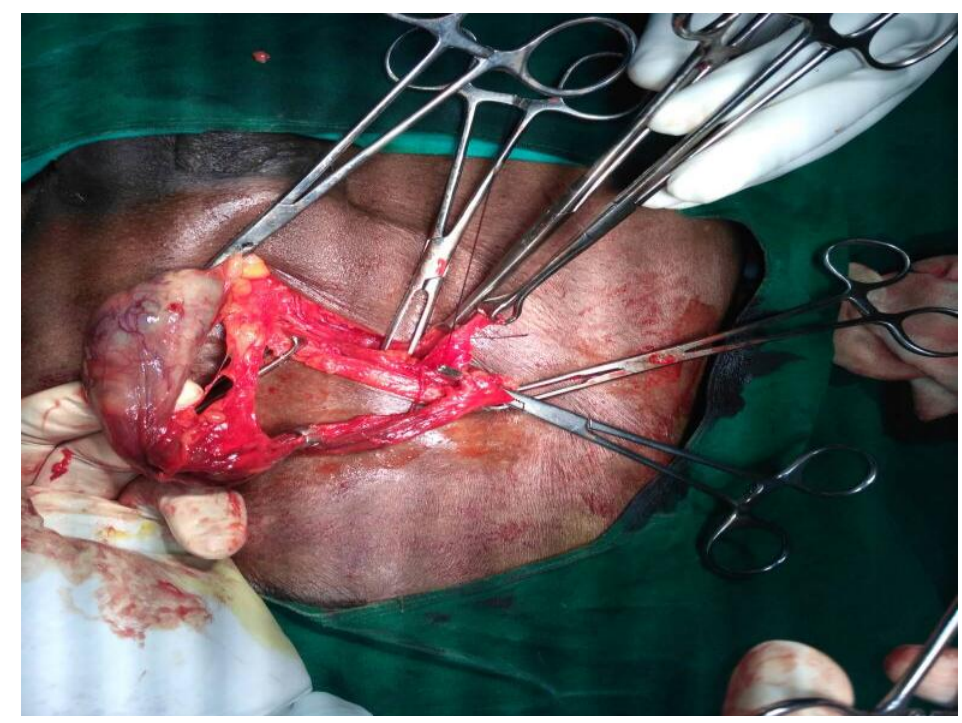

Fig 3: Gangrenous cord structures with the testes.

Direct hernias protrude medial to the inferior epigastric vessels, within Hesselbach's triangle. The borders of the triangle are the inguinal ligament inferiorly, the lateral edge of rectus sheath medially, and the inferior epigastric vessels superolaterally ${ }^{5}$. The contents of the abdominal cavity can descend into the hernia sac as a long-term process and they may be entangled within the hernia causing an intestinal obstruction. Hernia with these features but with preserved blood supply is called Obstructed Hernia. Hernia is associated with intestinal obstruction due to occlusion of the lumen of the bowel. A hernia is said to be Strangulated when the blood supply of its contents is impaired. The condition develops when the neck of the sac is very much constricted. Gangrenous changes may start as early as 5 to 6 hours after the onset of first symptom (pain) ${ }^{6}$. Strangulated external hernias account for $18-20 \%$ of all intestinal obstructions in adults ${ }^{78}$. Indirect inguinal hernias carry more risk of strangulation and incarceration than direct hernias. When they become incarcerated the incidence is about $34.1 \%$ versus $16.7 \%$ and $32.6 \%$ versus $10.3 \%$ in two different studies ${ }^{9}$. According to another study, the incidence of strangulation leading to bowel resection, in case of indirect and direct inguinal hernia was $32.1 \%$ and $11.8 \%$ respectively ${ }^{9}$. Even though the neck of the direct hernial sac (fascial defect) is soft and wide enough to avoid strangulation in the early stage of the hernia, it may become fibrotic, solid and narrowed with time. This process may create a risk for a direct hernia to be incarcerated ${ }^{9}$. We are reporting this case because, here a conventional laparotomy was not required. But on exploration the obstructed hernia was found to be a direct type with intestines not being involved and the gangrenous sac wall containing congested pre peritoneal pad of fat, cord structures, right testes.

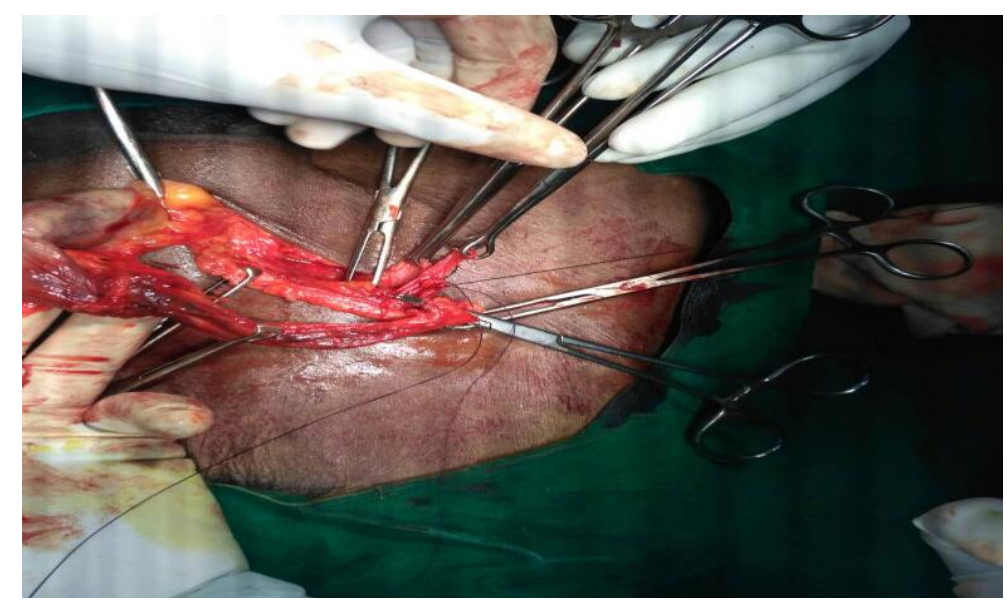

Fig 5: Excision of the cord structures with orchidectomy. 


\section{Conclusion}

Though incarceration and even strangulation are less common in direct inguinal hernia as compared to indirect inguinal hernia, a long standing direct inguinal hernia may present as acute or sub-acute intestinal obstruction especially in elderly patients. When in doubt it is always better to open and see. Though conventional laparotomy midline incision is not always required. Therefore, with high degree of suspicion we should repair direct inguinal hernias on an elective basis in any age group and never to be managed conservatively especially in elderly population.

\section{References}

[1]. Beauchamp CM, Ever BM, Mattox KL. Sabiston Textbook of Surgery: The biological basis of Modern Surgical Practice, 19ed. vol2. Philadelphia: Saunders; 2012. p. 1114

[2]. McIntosh A, Hutchinson A, Roberts A, Withers H. Evidence-based management of groin hernia in primary care--a systematic review. Fam Pract 2000 Oct;17(5):442-7.

[3]. Gould J. Laparoscopic versus open inguinal hernia repair. Surg Clin North Am 2008 Oct;88(5):1073-81,vii-viii.

[4]. Zinner MJ, Ashley SW. Maingot's Abdominal Operations, 12ed. New York: McGraw Hill Education; 2012. p. 124.

[5]. Brunicardi FC, Andersen DK, Billiar TR, et al. Schwartz's Principles Of Surgery, 10ed. New York: McGraw Hill Education; 2014. p. 1496.

[6]. S. DAS. A Text Book On Surgery, $9^{\text {th }}$ edition.

[7]. McEntee G, Pender D, Mulvin D, et al. Current spectrum of intestinal obstruction. Br J Surg 1987 Nov;74(11):976-80.

[8]. Kekec Y, Alparslan A, Demirtas S, et al. Effect of strangulation on morbidity and mortality in irreducible hernia. Turk J Surg 1993;9:128-31.

[9]. Kulacoglu H, Kulah B, Hatipoglu S. Coskun F. Incarcerated direct inguinal hernias: a three-year series at a large volume teaching hospital. Hernia 2000;4(3):145-7. 